\title{
Mosquito Gonotrophic Cycle and Multiple Feeding Potential: Contrasts Between Anopheles and Aedes (Diptera: Culicidae)
}

\author{
MARC J. KLOWDEN AND HANS BRIEGEL ${ }^{1}$ \\ Division of Entomology, University of Idaho, Moscow, ID 83844-2339
}

\begin{abstract}
J. Med. Entomol. 31(4): 618-622 (1994)
ABSTRACT Two aedine and three anopheline species were allowed to blood-feed to repletion, and their host-seeking behavior was measured with an olfactometer at intervals afterward. Both Aedes aegypti (L.) and Ae. albopictus (Skuse) were inhibited from subsequently seeking a host during the gonotrophic cycle, but Anopheles gambiae Giles, An. albimanus Wiedemann, and An. freeborni Aitken, all showed varying degrees of hostseeking behavior while their eggs matured. Continued host-seeking by some anophelines during the gonotrophic cycle has important implications for some assumptions commonly made about mosquito behavior and parasite transmission.
\end{abstract}

KEY WORDS Aedes, Anopheles, multiple feeding

THE LENGTH OF the mosquito gonotrophic cycle is among the most important of the bionomic parameters, because it is thought to be an estimate of the frequency at which the insects contact their hosts and, thus, an estimate of the opportunities for acquisition and transmission of parasites. The mosquito gonotrophic cycle, originally defined by Beklemishev (1940, cited in Detinova 1962), includes the search for a host and the ingestion of a blood meal, the digestion of the meal and the maturation of ovaries, and the laying of mature eggs after a search for an oviposition site. Attempts to estimate the average duration of the cycle in mosquito populations are based on both direct observations under laboratory conditions (Ramsey et al. 1988) and on field collection data that reflect the parity of populations (Charlwood et al. 1985, McHugh 1989). Field studies are based on several assumptions, including that the majority of females obtain only one blood meal during each cycle (Birley \& Rajagopalan 1981). Based on the length of the gonotrophic cycle, estimates of biting rates in field populations are used typically to incriminate a particular species in the transmission of parasites (Ijumba et al. 1990).

Once the duration of the gonotrophic cycle has been estimated, it is commonly assumed that host-seeking behavior is inhibited until eggs are laid, limiting the feeding frequency to once during each cycle (Garrett-Jones \& Shidrawi 1969, Roitberg \& Friend 1992), although field studies frequently have reported the occurrence of multiple meals during a single gonotrophic cycle (Edman \& Downe 1964, Boreham \& GarrettJones 1973, Boreham et al. 1979, Burkot et al.

${ }^{1}$ Department of Zoology, University of Zürich, CH-8057 Zürich, Switzerland.
1988). Because parasite transmission obviously can only occur when the mosquito feeds on a host, the assumptions that theoretically limit the occurrence of multiple feeding are important considerations in the biological equation that describes the vector efficiency of a species.

In laboratory-reared Aedes aegypti (L.), the failure to seek a host once the gonotrophic cycle has been initiated results from the operation of two distinct physiological mechanisms. Beginning with blood ingestion, distention-induced inhibition, which appears to be based on the activation of abdominal stretch receptors, acts until the blood meal has been digested and excreted and the abdominal distention reduced (Klowden \& Lea 1978, 1979b). If the blood meal initiates egg maturation, a second, humoral mechanism (oocyte-induced inhibition), then prevents host-seeking from continuing (Klowden \& Lea 1979a, Klowden 1981). Depending on the initial size of the blood meal and the nutritional state of the female (Klowden 1986), these two mechanisms often overlap to limit feeding frequency to once at the beginning of each gonotrophic cycle. Unfortunately, our insights into the existence of these mechanisms in other species besides Ae aegypti is limited mainly to Culex (Edman et al. 1975).

Briegel \& Rezzonico (1985) speculated that anophelines, which are primarily crepuscular and nocturnal feeders, may not have evolved as strong a mechanism for inhibiting host-seeking during the gonotrophic cycle as have day biting aedines. Defensive host behavior is a strong selective force (Edman \& Scott 1987) and may have been one of the selective pressures for the evolution of mechanisms that prevent host-seeking from occurring when it would not be biologically appropriate. Once an initial blood meal is in- 
gested and egg maturation is initiated by the mosquito, a second blood meal later during the gonotrophic cycle would not necessarily increase her fecundity (Lea et al. 1978), but might threaten her survival if the host is defensive (Edman et al. 1972). Natural selection would operate to eventually eliminate those insects that took risks that would be of no reproductive benefit. If host behavior has indeed contributed to the evolution of these mechanisms, we might expect nocturnal and crepuscular anopheline mosquitoes to have evolved less effective means of preventing host contact, because they feed on hosts during the period when the hosts are less defensive. The absence of a correlation between blood-feeding and egg development would undermine our reliance on the length of the ovarian cycle as an estimate of host contact for these insects.

In this current study, we compared the multiple feeding potential of two day-biting aedines, Ae. aegypti and Ae. albopictus (Skuse), with three crepuscular and evening-biting anophelines, Anopheles gambiae Giles, An. albimanus Weidemann, and An. freeborni Aitken, by determining their likelihood to resume host-seeking after they fed on blood to repletion. Thus, the recent findings on multiple blood meals taken by Anopheles (Briegel \& Hörler 1993) are extended from the metabolic to the behavioral level.

\section{Materials and Methods}

Laboratory colonies of Ae. aegypti, Ae. albopictus, An. albimanus, An. freeborni, and An. gambiae (Suakoko strain) were used in these experiments. Aedes larvae were reared at $27^{\circ} \mathrm{C}$ on a diet of brewer's yeast, lactalbumin hydrolysate, and finely ground rat chow (1:1:1 by weight). Anopheles larvae were reared at the same temperature on finely ground Tetramin (Tetra Werke, Germany) fish food. Adults were maintained at $27^{\circ} \mathrm{C}$, with $70-80 \% \mathrm{RH}$, and continuous access to $10 \%$ sucrose from cotton wicks. Mated females (4-5 d old) were used in the experiments. They were blood fed on a human host until they voluntarily withdrew their mouthparts. Immediately before blood-feeding took place at 0600 hours and then at various times afterwards, the host-seeking behavior of the mosquitoes was tested in the laboratory olfactometer described previously (Klowden \& Lea 1978). To measure host-seeking behavior, female mosquitoes were placed at one end of the apparatus, and human host stimuli introduced at the other end. Those mosquitoes that flew upwind through the $1-\mathrm{m}$ long flight chamber in response to a $10-\mathrm{min}$ exposure to host stimuli were considered to have engaged in host-seeking. Because mosquitoes that flew toward host stimuli also probed at the screening, it was assumed that these also would have fed on the host if given the chance. Indi-

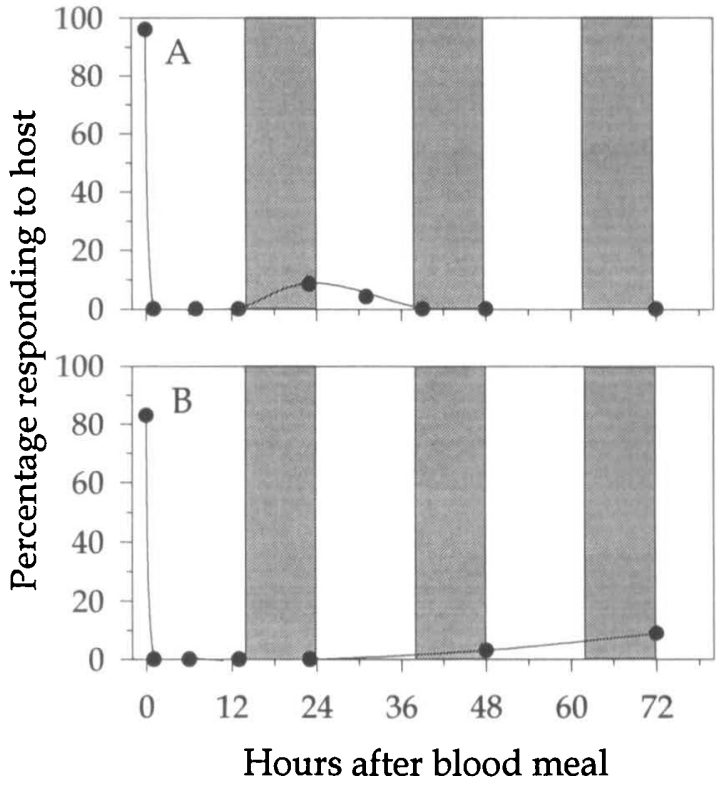

Fig. 1. Host-seeking responses of Ae. aegypti (A) and Ae. albopictus (B) to human host stimuli in a laboratory olfactometer. Each point represents the response of a different population of $30-50$ females. Shaded bars represent the scotophase.

vidual populations were tested only once; a profile of host-seeking as a function of time since blood ingestion was constructed based on several tests using a different population at each time interval.

For one experiment, mosquitoes were ovariectomized before they were allowed to blood feed. Unfed mosquitoes were placed within at small container, restrained with a strip of clay, and flooded with saline. Both ovaries were removed, using a sharpened forceps through a slit in the arthrodial membrane on the dorsal portion of the abdomen. Controls underwent a sham operation. The host-seeking responses of control, with intact ovaries, and experimental groups, with their ovaries removed, were compared at $48 \mathrm{~h}$ after a replete blood meal to determine the effect of ovarian development. The percentages that responded were transformed to arcsine values and their equality tested to determine statistical differences (Sokal \& Rohlf 1969).

\section{Results and Discussion}

Our data indicate that for some mosquito species, attempts to take multiple meals during a single gonotrophic cycle may be the rule. When treated to optimal nutritional conditions, laboratory-reared Ae. aegypti and Ae. albopictus both failed to seek a host after a replete blood meal was ingested (Fig. $1 \mathrm{~A}$ and B), an inhibition that continued until oviposition would have occurred. In contrast, although all three Anopheles 


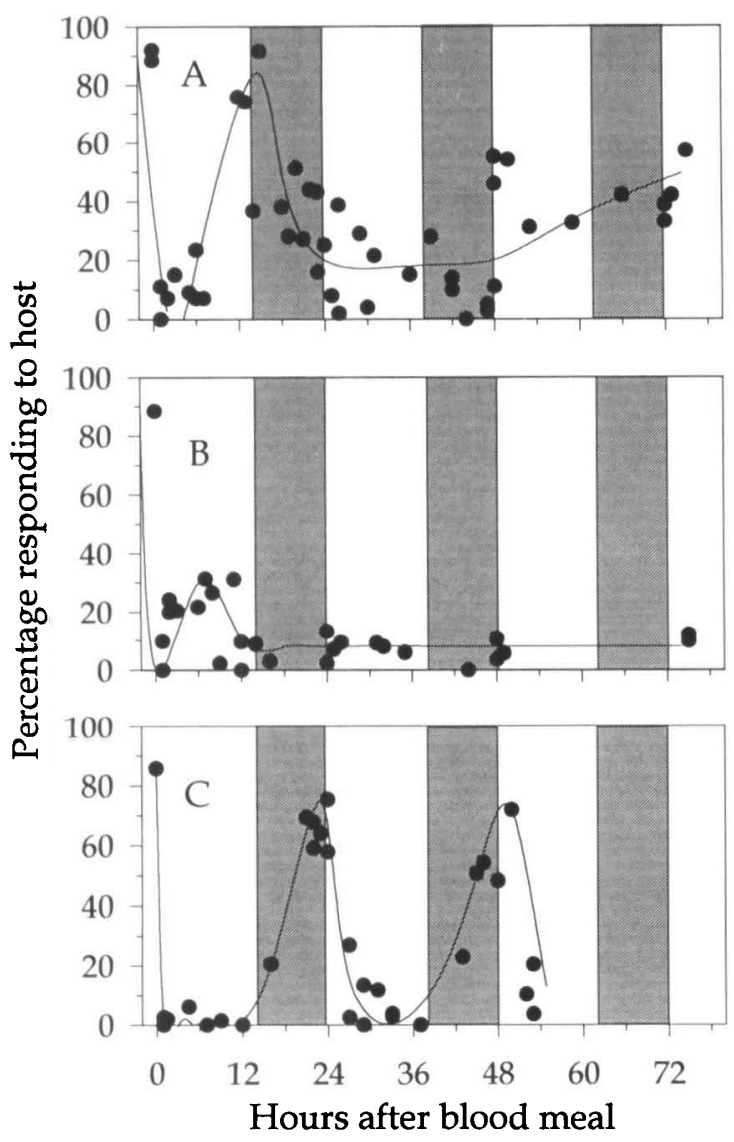

Fig. 2. Host-seeking responses of An. albimanus (A), An. freeborni (B) and An. gambiae (C). Each point represents the response of a different population of 30-50 females. Shaded bars represent the scotophase.

species generally responded to host stimuli immediately before blood ingestion, few responded immediately afterwards (Fig. $2 \mathrm{~A}-\mathrm{C}$ ), but showed variable responses to host stimuli. A small proportion of the An. albimanus population responded within $6 \mathrm{~h}$, and by 12-14 h their response was no different than before they had fed (Fig. 2A). As eggs developed, host-seeking declined but still occurred in a proportion of the population for the remainder of the gonotrophic cycle. The response of $A n$. freeborni populations was much reduced after blood ingestion, especially during egg development (Fig. 2B). Although An. gambiae were inhibited from seeking a host within $12 \mathrm{~h}$ of blood ingestion, they continued to respond according to a circadian rhythmicity toward the end of each scotophase (Fig. 2C).

The initial inhibition in all species appears to result from abdominal distention produced by blood ingestion (Klowden \& Lea 1979b). In the two Aedes species, this inhibition continues until a second mechanism of inhibition that is associated with egg maturation prevents host-
Table 1. Effects of ovariectomy on mosquito hostseeking behavior at $48 \mathrm{~h}$ after blood ingestion

\begin{tabular}{lcc}
\hline \multirow{2}{*}{ Mosquito species } & \multicolumn{2}{c}{$\%$ Seeking host $(n)$} \\
\cline { 2 - 3 } & Sham-operated controls & Ovariectomized \\
\hline Ae. aegypti & $0(26)$ & $79(24)^{*}$ \\
Ae. albopictus & $4(23)$ & $65(23)^{*}$ \\
An. albimanus & $20(25)$ & $80(20)^{*}$ \\
An. freeborni & $6(33)$ & $75(28)^{*}$ \\
\hline
\end{tabular}

$* P<0.05$.

seeking from occurring. Many Anopheles concentrate the blood meal during feeding to compensate for their relatively small midgut capacity (Briegel \& Rezzonico 1985), so the volume of blood they ingest is not as great as that of comparably-sized Aedes. Thus, the distentioninduced inhibition of host-seeking does not last as long and in the case of An. albimanus, a large proportion of the population exhibited a hostseeking response within $12 \mathrm{~h}$ (Fig. 2A). A small segment of the An. freeborni population also engages in host-seeking within $12 \mathrm{~h}$ after blood ingestion (Fig. 2B). Although few An. gambiae seek a host after an early morning blood meal, this inhibition was also characteristic of unfed females; the cyclic host-seeking behavior in this species is more rigidly tied to circadian rhythms and less to blood ingestion or egg development. The suppression of host-seeking behavior in all the species examined was related to egg maturation. When ovaries were removed, there was a significant increase in host-seeking behavior at $48 \mathrm{~h}$ following blood ingestion (Table $\mathrm{l}$ ). We did not examine the effects of ovaries in An. gambiae because this species showed no inhibition of host-seeking during oogenesis.

It must be emphasized that we measured the tendency for multiple feeding under ideal nutritional conditions in laboratory populations. Because host-seeking is suppressed to some degree during oogenesis, small blood meals that fail to trigger oogenesis, or even large meals ingested by nutritionally deficient mosquitoes that would not produce an egg batch, would fail to induce oocyte-induced, host-seeking inhibition. There have been several indications that more than one blood meal is necessary for egg development in field populations of both anophelines and aedines (Gillies 1954, 1955; Feinsod \& Spielman 1980), which Briegel \& Hörler (1993) explained on the basis of the caloric status of the females. Even when eggs do develop, the inhibition of host-seeking is strongly dependent on nutritional state; depriving female Ae. aegypti of carbohydrate significantly increased their hostseeking behavior during oogenesis (Klowden 1986). Thus, although the laboratory-reared Aedes failed to respond during the gonotrophic cycle in our experiments (Fig. $1 \mathrm{~A}$ and B), this does not necessarily imply that their behavior also is sup- 
pressed under field conditions. Field studies have demonstrated the occurrence of multiple feeding not only in populations of Anopheles (Senior-White 1952, Boreham \& Garrett-Jones 1973, Boreham et al. 1979, Burkot et al. 1988) but also in Ae. aegypti (Macdonald 1956, Sheppard et al. 1969, Scott et. al 1993).

There are at least two reasons for the differences in the host-seeking behavior of the two Aedes and the three Anopheles species we studied. If the defensive behavior of vertebrate hosts was the selective pressure for the evolution of mechanisms that inhibited host-seeking when it was no longer biologically appropriate, it is likely that the Anopheles species that feed at night have encountered less of this pressure by feeding on hosts when they were less defensive and thus had less to lose than did day-biting Aedes. In addition, metabolic differences between the two genera (Briegel 1990a, b) indicate that anophelines rely on a strategy of compensating for a smaller midgut capacity and reduced carryover of larval reserves by feeding multiply (Briegel \& Hörler 1993).

Although there have been numerous reports of multiple feeding behavior in both genera, our data indicate that the endogenous mechanisms that regulate anopheline behavior are different than in aedines, and under similar ecological conditions, they are probably more likely to engage in multiple feeding during a single gonotrophic cycle. This is an important consideration to include in models of mosquito-borne disease or attempts to determine the levels to which insect populations must be reduced to assure that transmission rates are reduced. Given that mosquito feeding is often independent of the ovarian cycle, relying strictly on the length of the gonotrophic cycle to assess feeding frequency will result in significant underestimates of its occurrence.

\section{Acknowledgments}

This investigation was supported in part by grants INT-8813376 from the National Science Foundation and AI-24453 from the National Institutes of Health to M.J.K. Contribution 93732 from the Idaho Agricultural Experiment Station.

\section{References Cited}

Beklemishev, W. N. 1940. [Gonotrophic rhythm as a basic principle of the biology of Anopheles]. Vopr. Fiziol. Ekol. Malar. Komara 1: 3-22 (cited in Detinova 1962).

Birley, M. H. \& P. K. Rajagopalan. 1981. Estimation of the survival and biting rates of Culex quinquefasciatus (Diptera: Culicidae). J. Med. Entomol. 18: 81-186.

Boreham, P.F.L. \& C. Garrett-Jones, 1973. Prevalence of mixed blood meals and double feeding in a malaria vector (Anopheles sacharovi Favre). Bull. W.H.O. 48: 605-614.

Boreham, P.F.L., J. K. Lenahan, R. Boulzaquet, J. Storey, T. S. Ashkar, R. Nambiar \& T. Matsushima. 1979. Studies on multiple feeding by Anopheles gambiae s.l. in a Sudan savanna area of north Nigeria. Trans. R. Soc. Trop. Med. Hyg. 73: 418-423.

Briegel, H. 1990a. Metabolic relationship between female body size, reserves, and fecundity of Aedes aegypti. J. Insect Physiol. 36: 165-172.

1990b. Fecundity, metabolism, and body size in Anopheles (Diptera: Culicidae), vectors of malaria. J. Med. Entomol. 27: 839-850.

Briegel, H. \& E. Hörler. 1993. Multiple blood meals as a reproductive strategy in Anopheles (Diptera: Culicidae). J. Med. Entomol. 30: 975-985.

Briegel, H. \& L. Rezzonico. 1985. Concentration of host blood protein during feeding by Anopheles mosquitoes (Diptera: Culicidae). J. Med. Entomol. 22: 612-618.

Burkot, T. R., P. M. Graves, R. Paru \& M. Lagog. 1988. Mixed blood feeding by the malaria vectors in the Anopheles punctulatus complex (Diptera: Culicidae). J. Med. Entomol. 25: 205-213.

Charlwood, J. D., M. H. Birley, H. Dagoro, R. Paru \& R. R. Holmes. 1985. Assessing survival rates of Anopheles farauti (Diptera: Culicidae) from Papua New Guinea. J. Anim. Ecol. 54: 1003-1016.

Detinova, T. S. 1962. Age grouping methods in Diptera of medical importance. W.H.O. Monograph Ser. No. 47: 216.

Edman, J. D., E. Cody \& H. Lynn. 1975. Bloodfeeding activity of partially engorged Culex nigripalpus (Diptera: Culicidae). Entomol. Exp. Appl. 18: 261-268.

Edman, J. D. \& A.E.R. Downe. 1964. Host blood sources and multiple feeding habits of mosquitoes in Kansas. Mosq. News 24: 154-160.

Edman, J. D. \& T. W. Scott. 1987. Host defensive behavior and the feeding success of mosquitoes. Insect Sci. Appl. 8: 617-622.

Edman, J. D., L. A. Webber \& H. W. Kale, II. 1972. Effect of mosquito density on the interrelationship of host behavior and mosquito feeding success. Am. J. Trop. Med. Hyg. 21: 487-491.

Feinsod, F. M. \& A. Spielman. 1980. Nutrientmediated juvenile hormone secretion in mosquitoes. J. Insect Physiol. 26: 113-117.

Garrett-Jones, C. \& G. R. Shidrawi. 1969. Malarial vectorial capacity of a population of Anopheles gambiae. An exercise in epidemiological entomology. Bul]. W.H.O. 40: 531-545.

Gillies, M. T. 1954. The recognition of age-groups within populations of Anopheles gambiae by the pre-gravid rate and the sporozoite rate. Ann. Trop. Med. Parasitol. 48: 58-74.

1955. The pre-gravid phase of ovarian development in Anopheles funestus. Ann. Trop. Med. Parasitol. 49: 320-325.

Ijumba, J. N., R. W. Mwangi \& J. C. Beier. 1990. Malaria transmission potential of Anopheles mosquitoes in the Mwea-Tebere irrigation scheme, Kenya. Med. Vet. Entomol. 4: 425-432.

Lea, A. O., H. Briegel \& H. M. Lea. 1978. Arrest, resorption, or maturation of oöcytes in Aedes aegypti: dependence on the quantity of blood and the interval between blood meals. Physiol. Entomol. 3: 309-316. 
Klowden, M. J. 1981. Initiation and termination of host-seeking inhibition in Aedes aegypti during oocyte maturation. J. Insect Physiol. 27: 799-803.

Klowden, M. J. \& A. O. Lea. 1978. Blood meal size as a factor affecting continued host-seeking by Aedes aegypti (L.). Am. J. Trop. Med. Hyg. 27: 827831.

1979a. Humoral inhibition of host-seeking in Aedes aegypti (L.) during oocyte maturation. J. Insect Physiol. 25: 231-235.

1979b. Abdominal distention terminates subsequent host-seeking behavior of Aedes aegypti following a blood meal. J. Insect Physiol. 25: 583-585.

Macdonald, W. W. 1956. Aëdes aegypti in Malaya. II. Larval and adult biology. Ann. Trop. Med. Parasitol. 50: 399-414.

McHugh, C. P. 1989. Ecology of a semi-isolated population of adult Anopheles freeborni: abundance, trophic status, parity, survivorship, gonotrophic cycle length, and host selection. Am. J. Trop. Med. Hyg. 41: 169-176.

Ramsey, J. M., E. Salinas, J. R. Lopez, G. AngelCabañas, L. Martinez \& D. N. Brown. 1988. Laboratory oviposition, fecundity and egg hatching ability of colonized Anopheles albimanus from southwestern Mexico. J. Am. Mosq. Contr. Assoc. 4: 509-515.

Roitberg, B. D. \& W. G. Friend. 1992. A general theory for host-seeking decisions in mosquitoes. Bull. Math. Biol. 54: 401-412.

Scott, T. W., G. G. Clark, L. H. Lorenz, P. H. Amerasinghe, P. Reiter \& J. D. Edman. 1993. Detection of multiple blood feeding in Aedes aegypti (Diptera: Culicidae) during a single gonotrophic cycle using a histologic technique. J. Med. Entomol. 30: 94-99.

Senior-White, R. A. 1952. Studies on the bionomics of Anopheles aquasalis Curry, 1932 (concld). Part III. Indian J. Malariol. 6: 29-72.

Sheppard, P. M., W. W. Macdonald, R. J. Tonn \& B. Grab. 1969. The dynamics of an adult population of Aedes aegypti in relation to dengue haemorrhagic fever in Bangkok. J. Anim. Ecol. 38: 661697.

Sokal, R. R. \& F. J. Rohlf. 1969. Biometry. W. H. Freeman, San Francisco.

Received for publication 21 September 1993; accepted 7 March 1994. 